ARTIGO ORIGINAL

\title{
Avaliação do teor ótimo de resinas orgânicas para produção de painéis OSB de madeira Balsa (Ochroma pyramidale) residual
}

\author{
Evaluation of the optimum content of organic resins for the production of \\ residual OSB wood of Balsa (Ochroma pyramidale) panels
}

\author{
Wanley Eduardo Lopes Junior ${ }^{1}$ (D), Guilherme Henrique Ament Barbirato ${ }^{1}$ (D), Mariana Pavesi ${ }^{1}$ (D), \\ Julio Soriano ${ }^{2}$ (D) , Juliano Fiorelli ${ }^{1}$ (B) \\ ${ }^{1}$ Universidade de São Paulo - USP. Pirassununga, SP, Brasil \\ 2 Universidade Estadual de Campinas - UNICAMP. Campinas, SP, Brasil
}

Como Citar: Lopes Junior, W. E., Barbirato, G. H. A., Pavesi, M., Soriano, J., Fiorelli, J. (2021). Avaliação do teor ótimo de resinas orgânicas para produção de painéis OSB de madeira balsa (Ochroma Pyramidale) residual. Scientia Forestalis, 49(129), e3442. https://doi.org/10.18671/scifor.v49n129.22

\begin{abstract}
Resumo
Pesquisas realizadas com enfoque no desenvolvimento e avaliação de painéis OSB de madeira Balsa (Ochroma Pyramidale) residual não definiram o teor ótimo de resina orgânica. Sendo assim, este trabalho teve como objetivo avaliar diferentes teores de resinas orgânicas poliuretana à base de óleo de mamona, fenol-formaldeído e ureia-formaldeído em painéis de partículas orientadas (OSB) de madeira Balsa residual. Para atingir os objetivos foram produzidos painéis OSB com partículas do tipo strand, densidade de $650 \mathrm{~kg} / \mathrm{m}^{3}$, espessura $10 \mathrm{~mm}$ e teores de resinas 13\%, 15\% e 18\%. Esses painéis foram caracterizados pelas propriedades físicas e mecânicas seguindo diretrizes de normativas internacionais. Os resultados obtidos indicaram que os painéis produzidos com $13 \%$ de resina poliuretana à base de óleo de mamona e fenol-formaldeído apresentaram melhor desempenho físico e mecânico e foram classificados, segundo recomendações da EN 300:2002, como painéis OSB do tipo 1, com indicativo de usos gerais, incluindo-se mobiliário e aplicações em ambientes internos, sem contato com fontes de umidade. A resina ureiaformaldeído nos teores estudados não apresentou desempenho físico e mecânico que atenda às recomendações da EN300:2002.
\end{abstract}

Palavras Chaves: Painel de partículas orientadas; Madeira residual; Propriedades físicas e mecânicas.

\begin{abstract}
Research focused on the development and evaluation of residual OSB wood panels of Balsa (Ochroma Pyramidale) did not define the optimum organic resin content. Thus, this study aimed to evaluate different contents of organic resins (polyurethane based on castor oil, phenol-formaldehyde and ureaformaldehyde) in particle boards oriented (OSB) of residual Balsa wood. To achieve the objectives OSB panels with strand-type particles, density of $650 \mathrm{~kg} / \mathrm{m}^{3}$, thickness of $10 \mathrm{~mm}$ and three resin contents $(13 \%$, $15 \%$ and $18 \%$ ) were produced. These panels were characterized by physical and mechanical properties following the guidelines of international norms. The results indicated that the panels produced with $13 \%$ of polyurethane resin based on castor oil and phenol-fomaldehyde showed better physical and mechanical performances and were classified according to recommendations of EN300:2002, as OSB panels of Type 1, with indication for general uses, including furniture and for indoor environments, without contact with moisture sources. The urea-formaldehyde resin at the studied contents did not present a physical and mechanical performance that meets the recommendations of the EN300:2002.
\end{abstract}

Keywords: Particle oriented panel; Residual wood; Physical and mechanical properties.

Fonte de financiamento: FAPESP (proc. 2017/18076-4) e CNPq (proc. 407451/2018-8 e proc. 304106/2017-8). Este estudo foi financiado em parte pela coordenação de aperfeiçoamento de pessoal de nível superior-Brasil (CAPES) - código financeiro 001.

Conflito de interesse: Nada a declarar.

Autor correspondente: wanley.lopes@usp.br

Recebido: 12 agosto 2019.

Aceito: 26 março 2020

Editor: Paulo Henrique Müller Silva.

(c) Este é um artigo publicado em acesso aberto (Open Access) sob a licença Creative Commons Attribution, que permite uso, distribuição e gy reprodução em qualquer meio, sem restrições desde que o trabalho original seja corretamente citado. 


\section{INTRODUÇÃO}

O termo biomassa florestal primária (BFP) pode ser definido como a parcela biodegradável no processo de geração de novos produtos com fins energéticos. Em alguns casos, como reflorestamento, a BFP tem sua origem nos materiais vegetais em operações de manejo, dentre elas: podas, desbastes, seleção de brotos, plantas pequenas, e controle da vegetação. Já a biomassa florestal secundária (BFS) é formada por matéria orgânica residual, como: cavacos, pedaços, serragem, entre outros, podendo ser encontrada em processos da indústria de transformação da madeira. Entretanto, constata-se a presença de BFS em resíduos urbanos da construção civil, de outras atividades industriais (pallets e embalagens) e das podas (Congresso Internacional de Biomassa, 2018).

O destino desse material é discutido em vários trabalhos científicos com propostas alternativas para a queima da biomassa florestal utilizada para a produção de energia (Mendes et al., 2010; Cravo et al., 2015; Christoforo et al., 2015). Entretanto, diversas pesquisas desenvolvidas nos últimos anos, em diferentes regiões do mundo, têm explorado o uso de matéria-prima fibrosa oriunda de subprodutos agroindustriais ou da biomassa florestal, para a produção de painéis de partículas aglomerados com resina orgânica ou inorgânica, os quais apresentam resultados que permitem sua aplicação em diferentes áreas (Khalil et al., 2010; Mendes et al., 2010; Ortunõ et al., 2011; Varanda et al., 2014; Ronquim et al., 2014; Cravo et al., 2015; Christoforo et al., 2015; Macedo et al., 2016), agregando valor ao resíduo e proporcionando um destino mais sustentável para essa matéria prima.

Dentre esses subprodutos tem-se a madeira Balsa (Ochroma Pyramidale), que segundo Daniels (2017) é uma madeira de baixa densidade $\left(120 \mathrm{~kg} / \mathrm{m}^{3}\right.$ a $\left.200 \mathrm{~kg} / \mathrm{m}^{3}\right)$, possui elevada taxa de crescimento variando de 18 a 25 metros de altura em aproximadamente 7 anos (Finger et al., 2012) e, propriedades mecânicas que podem atingir 8 GPa de módulo de elasticidade e $70 \mathrm{MPa}$ de módulo de ruptura na flexão estática (Borrega et al., 2015).

Os painéis OSB podem ser um dos destinos desses subprodutos, uma vez que em sua produção são utilizadas partículas de madeira de baixa qualidade (toras finas e tortas, galhadas, cavacos e em alguns casos a casca) (Cloutier, 1998; Bortoletto Júnior \& Garcia, 2004).

O adesivo é outra variável de fundamental importância na produção de painéis de partículas devido às implicações técnicas e econômicas, uma vez que a resina pode representar 30 a $60 \%$ do custo da produção e apresenta estreita relação com as propriedades mecânicas e de estabilidade dimensional (Barros Filho, 2009).

Em pesquisa recente, Barbirato et al. (2018) apresentaram um estudo de painéis de partículas orientadas (OSB) a partir de resíduos de madeira Balsa aglomeradas com resina poliuretana à base de óleo de mamona. Os autores avaliaram painéis com diferentes densidades $\left(300 \mathrm{~kg} / \mathrm{m}^{3}\right.$ e $400 \mathrm{~kg} / \mathrm{m}^{3}$ ) em diferentes teores (11\% e 15\%). Os resultados obtidos indicam que os painéis com densidade de $400 \mathrm{~kg} / \mathrm{m}^{3}$ e com teor de $15 \%$ de resina atingiram as exigências da Norma EN 300:2002, OSB tipo 1. No entanto, o teor de resina orgânica de $15 \%$ é considerado elevado pelo setor em que se insere o produto. Assim, o refinamento desse teor de resina em painéis OSB de madeira Balsa residual é um campo a ser investigado.

Sendo assim, o objetivo do trabalho consistiu em definir o melhor teor de resina poliuretana à base de óleo de mamona, fenol-formaldeído e ureia-formaldeído para produzir painéis OSB com madeira Balsa residual.

\section{MATERIAIS E MÉTODOS}

Para o desenvolvimento deste trabalho os resíduos de madeira Balsa coletados na indústria de processamento de madeira foram caracterizados visando identificar o potencial de uso como partículas para produção de painéis OSB. Posteriormente, foram produzidos e avaliados painéis OSB com partículas de madeira Balsa residual aglomeradas com 13, 15 e $18 \%$ de resinas poliuretana à base de óleo de mamona, ureia-formaldeído e fenolformaldeído. O teor superior das resinas orgânicas avaliadas neste estudo em comparação ao utilizado industrialmente $(8-11 \%)$ se deve a baixa densidade da madeira Balsa, maior 
porosidade, vasos com diâmetros maiores e com isso consequentemente, ao volume de partículas necessário para se atingir a densidade almejada $\left(650 \mathrm{Kg} / \mathrm{m}^{3}\right)$ para o painel quando comparado com painéis produzidos industrialmente com madeiras comerciais de Pinus e Eucalipto.

\section{Caracterização da madeira Balsa residual}

A madeira Balsa residual foi caracterizada por ensaios físicos (densidade aparente), químicos ( $\mathrm{pH}$ e composição química: celulose, hemicelulose e lignina) e anatômicos (imagens de microscopia eletrônica de varredura - MEV) da superfície e da seção transversal. Com o intuito de determinar a densidade aparente das partículas de madeira balsa foi utilizado a metodologia proposta por Moura \& Figueiredo (2002). Para determinação do pH da matéria prima foi realizada a metodologia adaptada de Vital (1973) e para composição química foi adotada a metodologia descrita por Van Soest (1994) e Empresa Brasileira de Pesquisa Agropecuária (2010) a fim de quantificar os teores de celulose, hemicelulose e lignina do material. Com o MEV foi possível analisar a anatomia dos poros e espessura da parede das fibras a fim de verificar o potencial da madeira Balsa no processo de aglomeração das partículas e da dispersão da resina.

\section{Produção de painéis OSB}

Os painéis OSB foram produzidos com partículas de madeira Balsa residual seguindo as seguintes etapas: 1- a madeira balsa residual foi processada em picador de lasca (marca Marconi, modelo MA685) com acionamento por motor elétrico, sendo geradas lascas com $90 \mathrm{~mm}$ de comprimento, $25 \mathrm{~mm}$ de largura e $1 \mathrm{~mm}$ de espessura (Figura 1A); 2- essas partículas foram remetidas para uma estufa com temperatura de $65^{\circ} \mathrm{C}$, por 48 horas, para se obter um material com $8 \%$ de umidade; 3- aplicação da resina por aspersão; 4- o material foi inserido em um molde formador de colchão (400 mm x $400 \mathrm{~mm} \times 10 \mathrm{~mm}$ ) levando em consideração a proporção face-miolo-face de massa igual a 30:40:30 (Figura 1B) e transferido para a prensa termo-hidráulica (pressão $50 \mathrm{kgf} / \mathrm{cm}^{2}$, temperatura de $100^{\circ} \mathrm{C}$ quando usado resina $\mathrm{PU}$ de mamona, $110^{\circ} \mathrm{C}$ para resina $\mathrm{UF}, 140^{\circ} \mathrm{C}$ para resina $\mathrm{FF}$ e tempo de prensagem de 10 minutos).

Com o fim do processo de prensagem, os painéis foram armazenados em temperatura ambiente por $72 \mathrm{~h}$, período marcado pela continuação do processo de cura da resina. Após esse período, os painéis foram refilados em dimensões finais $(400 \mathrm{~mm} \times 400 \mathrm{~mm}$ ) dos quais foram extraídos corpos de prova para a condução dos ensaios de caracterização do material.

Assim, foram fabricados painéis (Figura 1C) de média densidade $\left(650 \mathrm{~kg} / \mathrm{m}^{3}\right)$, com resina poliuretana à base de óleo de mamona (PU de mamona), fenol-formaldeído (FF) e ureiaformaldeído (UF), em diferentes teores (13\%, 15\% e 18\%) em massa de partículas, com espessura de $10 \mathrm{~mm}$, conforme tratamentos apresentados na Tabela 1, totalizando 12 painéis. A resina PU de Mamona utilizada era bicomponente, com viscosidade média de 167 CPs formulado pela mistura a frio de um pré-polímero (componente A) com 99,92\% de teor de sólidos e um poliol (componente B) com 99,91\% de teor de sólidos (praticamente isento de solventes). A resina FF utilizada tinha 70\% de teor de sólidos, viscosidade entre 200 - 450 cPs, $\mathrm{pH}$ entre $8,5-9,5$ e densidade entre $1,22-1,26 \mathrm{~g} / \mathrm{cm}^{3}$. A resina UF utilizada, possuía teor de sólidos entre 63,5 - 66\%, viscosidade entre 100 - 300 cPs, pH entre 7,5 - 8,2 e densidade entre $1,26-1,30 \mathrm{~g} / \mathrm{cm}^{3}$.

\section{Caracterização dos painéis OSB de madeira Balsa residual}

Os painéis OSB de madeira Balsa residual foram caracterizados por meio de ensaios mecânicos (flexão estática e tração perpendicular) e físico (Inchamento em espessura) seguindo as recomendações do European Committee for Standardization (1993a, 1993b, 1993c) com as respectivas normas: EN 310:2000 - Wood-based panels: Determination of modulus of elasticity in bending and of bending strength; EN-317:1993 - Particleboards and fibreboards - 
Determination of swelling in thickness after immersion in water e EN-319:1993 - Particleboards and fibreboards - Determination of tensile strength perpendicular to the plane of the board.

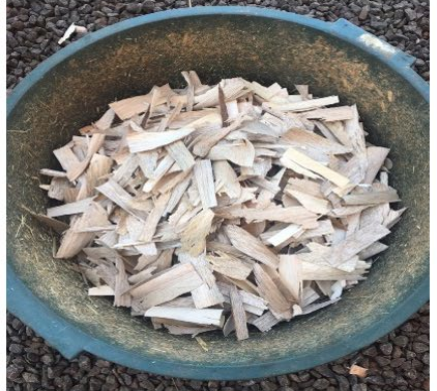

(A)

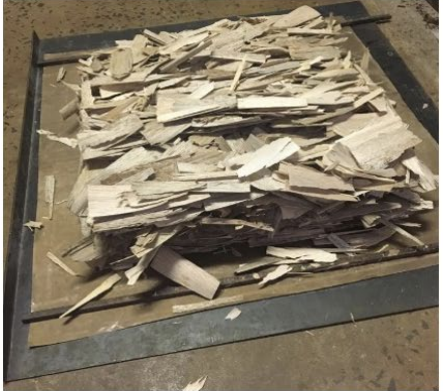

(B)

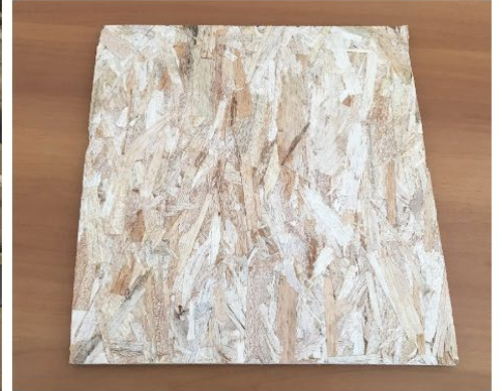

(C)

Figura 1: Processo de produção dos painéis OSB. A) Lascas ("strand") de madeira balsa. B) Colchão de partículas orientadas. C) Painel de partícula OSB de madeira Balsa.

Tabela 1. Plano experimental

\begin{tabular}{ccc}
\hline Tratamento & Resina & Teor (\%) \\
\hline T1-M & & 13 \\
T2-M & PU de Mamona & 15 \\
T3-M & & 18 \\
\hline T1-FF & & 13 \\
T2-FF & FF & 15 \\
T3-FF & & 18 \\
T1-UF & & 13 \\
T2-UF & UF & 15 \\
T3-UF & & 18 \\
\hline
\end{tabular}

Os resultados foram comparados com as indicações de classes estabelecidas pelo European Committee for Standardization (2002) com a norma EN300:2002 - Oriented Strand Boards (OSB) - Definitions, classification and specifications, a fim de verificar a qualidade dos materiais frente às diferentes possibilidades de uso na indústria moveleira, bem como na construção civil.

\section{Análise Estatística}

Os valores obtidos para as propriedades físico-mecânicas foram avaliados pela estatística descritiva, com o propósito de organizar os resultados. Como medida de tendência central foi adotada a média aritmética e, como medida de dispersão, o coeficiente de variação.

Após a análise descritiva, os dados foram submetidos a uma análise inferencial para diagnosticar a existência de diferença significativa entre os tratamentos estudados. Foi realizado um delineamento inteiramente casualizado (DIC) e os dados comparados pelo teste de comparação múltipla (Tukey) quando a ANOVA foi significativa, sendo ambos testados a $p<0,05$, devido ao cruzamento dos dados serem realizados individualmente para cada tipo de resina tendo como tratamento os teores de resina $(13,15$ e 18\%) em virtude das diferentes características dos adesivos analisados. 


\section{RESULTADOS E DISCUSSÃO}

Os resultados obtidos dos procedimentos experimentais contemplam as características da matéria prima e dos painéis OSB de partículas de madeira Balsa residual aglomeradas com resina PU de mamona, UF e FF.

\section{Propriedades físicas, químicas e anatômicas da madeira Balsa residual}

A matéria prima caracterizada para a confecção dos painéis mostrou para o resíduo da madeira Balsa uma baixa densidade aparente $\left(210 \mathrm{~kg} / \mathrm{m}^{3}\right)$ e, dentre os três constituintes elementares a preponderância da celulose (Tabela 2).

Tabela 2. Valores médios de propriedades físicas e químicas da madeira Balsa residual e outras madeiras

\begin{tabular}{|c|c|c|c|c|c|c|}
\hline Amostra & $\begin{array}{c}\text { Densidade } \\
\text { Aparente } \\
\left(\mathrm{kg} / \mathrm{m}^{3}\right)\end{array}$ & $\mathrm{pH}$ & $\begin{array}{c}\text { Celulose } \\
(\%)\end{array}$ & $\begin{array}{c}\text { Hemicelulose } \\
(\%)\end{array}$ & $\begin{array}{l}\text { Lignina } \\
(\%)\end{array}$ & Fonte \\
\hline Balsa & 210 & 5,44 & 69,03 & 10,50 & 10,39 & Estudo \\
\hline Pinus spp. & $560 *$ & 4,48 & 51,13 & 15,10 & 27,29 & $\begin{array}{l}\text { Fiorelli et al. } \\
\qquad(2014)\end{array}$ \\
\hline $\begin{array}{c}\text { Eucalyptus } \\
\text { urophylla } \\
\text { (Timor) }\end{array}$ & $510 * *$ & --- & 52,00 & 19,04 & 28,60 & $\begin{array}{l}\text { Megaton et al. } \\
\text { (2006) }\end{array}$ \\
\hline $\begin{array}{c}\text { Eucalyptus } \\
\text { grandis }\end{array}$ & $620 * *$ & --- & 51,70 & 20,50 & 27,80 & $\begin{array}{l}\text { Megaton et al. } \\
(2006)\end{array}$ \\
\hline
\end{tabular}

* Pinus Elliotti - Fonte: Associação Brasileira de Normas Técnicas (1997): NBR7190-1997. **Fonte: Brito \& Barrichelo (1977).

Ao comparar a densidade aparente das partículas de madeira Balsa residual com as densidades das espécies de madeiras utilizadas industrialmente na produção de painéis OSB (Tabela 2), observa-se que a madeira estudada possui densidade inferior. $\mathrm{O} \mathrm{pH}$, ácido, é similar ao Pinus spp. Em comparação às madeiras de Pinus spp. e Eucalyptus, a composição química das partículas de madeira Balsa tem teores superiores de celulose e inferiores de hemicelulose e lignina.

Segundo D'Almeida (1988) a celulose e hemicelulose apesar das semelhanças, diferem entre as suas reatividades, fato em que as hemiceluloses pelas configurações irregulares e ramificadas absorvem água mais facilmente que a celulose. Fengel \& Wegener (1984) defendem que o alto conteúdo de celulose aumenta a resistividade da madeira, enquanto o alto teor de lignina melhora a rigidez, conferindo melhores propriedades mecânicas aos painéis. A lignina é um componente que atua como agente selante por isso é uma propriedade aglomerante de extrema importância, ou seja, altos teores de lignina ocasionam uma melhora na aglomeração das partículas nos painéis. Como visto na Tabela 2, o teor de lignina na madeira Balsa estudada mostrou-se inferior quando comparado às outras madeiras comerciais, o que reflete a necessidade de maiores teores de resina para garantir uma adequada aglomeração das partículas.

Além dos baixos teores de lignina, vale destacar que a madeira Balsa apresentou baixa densidade e aproximada concentração de outros componentes como, por exemplo, a celulose e a hemicelulose, em relação as espécies de madeiras utilizadas comercialmente na produção de OSB, fato que a torna uma madeira com potencial para uso na produção desse tipo de painel.

\section{Características anatômicas da madeira Balsa residual}

Pela composição da estrutura anatômica de uma amostra de madeira Balsa residual, na seção transversal com aumento de 1.500x e 200x, Figura 2, foi possível visualizar, 
respectivamente os diâmetros dos poros, a espessura das paredes fibrilares e o diâmetro dos vasos.

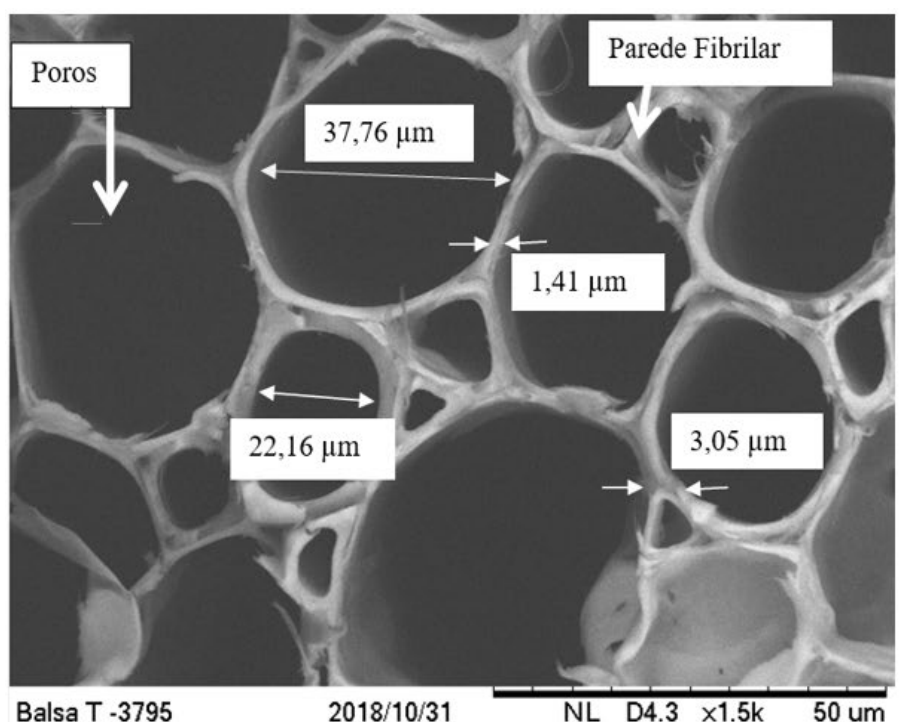

(A)

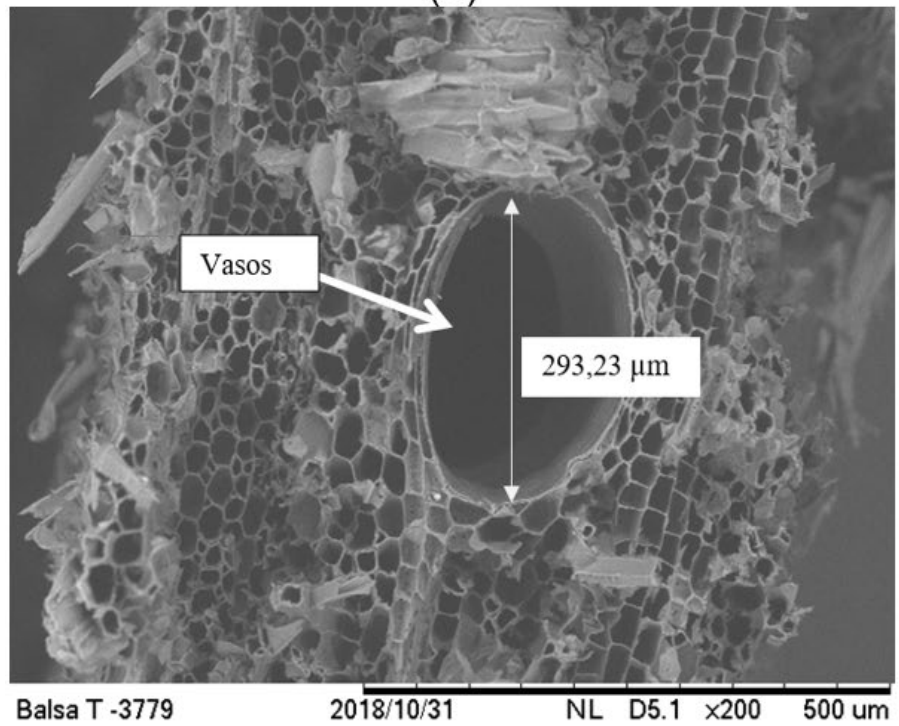

(B)

Figura 2 - Corte transversal de partícula de madeira Balsa residual. (A) Diâmetro dos poros e espessura das paredes fibrilares (imagem ampliada 1.500x). (B) Diâmetro dos vasos (imagem ampliada 200x).

Na Figura 2A, foram mensurados os valores dos diâmetros dos poros das partículas de madeira Balsa residual. A espessura das paredes fibrilares varia de 1,4 a 3,05 $\mu \mathrm{m}$. Fiorelli et al. (2014) identificaram através da microscopia eletrônica de varredura que o diâmetro dos poros e a espessura das paredes fibrilares para madeira Pinus spp. é em média $5 \mu \mathrm{m}$. Essa dimensão da espessura fibrilar da madeira Balsa residual, inferior a madeira de Pinus spp., a caracteriza como uma espécie de madeira moldável, garantindo assim maior superfície de contato entre as partículas e, consequentemente, resulta em painéis OSB com propriedades mecânicas compatíveis com painéis comerciais.

A Figura 2B apresenta as fibrilas e os vasos fibrilares cuja anatomia é característica para partículas de material com baixa densidade, indicando que a alta porosidade das partículas possibilitará a dispersão da resina durante a conformação do painel OSB. Easterling et al. (1982), Da Silva \& Kyriakides (2007) e Borrega et al. (2015) também comprovaram esta alta porosidade da madeira Balsa com diâmetro de vasos variando de 200 a $350 \mu \mathrm{m}$. 


\section{Propriedades física e mecânicas dos painéis OSB de madeira Balsa residual}

As propriedades física e mecânicas dos painéis OSB de madeira Balsa residual obtidas de painéis confeccionados conforme os tratamentos da Tabela 1, são apresentadas pela média e respectivo coeficiente de variação (CV). Esses resultados obtidos experimentalmente foram discutidos tendo por base as especificações da norma europeia EN 300:2002.

\section{Painéis OSB com resina PU de Mamona}

Para cada um dos tratamentos com resina PU de mamona foram realizadas 8 repetições, com os resultados médios apresentados na Tabela 3, obtidos de acordo com a EN 317 os valores de Inchamento em espessura (IE), com a EN 310 os módulos de ruptura (MOR) e de elasticidade (MOE) e, pela EN 319 a adesão interna (AI).

Tabela 3. Valores médios das propriedades físicas e mecânicas de painéis OSB com resina PU de mamona

\begin{tabular}{|c|c|c|c|c|c|c|}
\hline \multirow{2}{*}{ Tratamento } & \multirow{2}{*}{$\frac{\text { IE }(\%)}{24 \text { horas }}$} & \multicolumn{2}{|c|}{ MOR (MPa) } & \multicolumn{2}{|c|}{ MOE (MPa) } & \multirow{2}{*}{ Al(MPa) } \\
\hline & & Long. & Trans. & Long. & Trans. & \\
\hline T1-M & $23,26^{b}$ & $34,00^{a}$ & $12,79^{b}$ & $5668^{a}$ & $1281^{b}$ & $0,33^{b}$ \\
\hline (CV) & 28,09 & 25,39 & 23,44 & 19,02 & 14 & 12,96 \\
\hline T2-M & $17,41^{\mathrm{ab}}$ & $21,98^{b}$ & $19,88^{a}$ & $4340^{b}$ & $1882^{a}$ & $0,49^{a}$ \\
\hline (CV) & 39,28 & 27,95 & 11,39 & 22,13 & 9,91 & 27,12 \\
\hline T3-M & $11,78^{a}$ & $32,52^{a}$ & $15,74^{b}$ & $4924^{\mathrm{ab}}$ & $1399^{b}$ & $0,43^{\mathrm{ab}}$ \\
\hline (CV) & 38,72 & 24,57 & 27,15 & 18,84 & 24,8 & 17,2 \\
\hline EN 300:2002 Tipo 1 & 25 & 20 & 10 & 2500 & 1200 & 0,3 \\
\hline
\end{tabular}

*Médias seguidas por letras diferentes na coluna diferem significativamente a 5\% pelo Teste Tukey

Na propriedade física de inchamento em espessura, foi observado que os tratamentos T2-M e T3-M não apresentaram diferença estatística entre si (pelos resultados compartilhados pelas letras ab e a), o mesmo ocorre em T1-M e T2-M (pelo compartilhamento b e ab), porém, T1-M e T3-M diferem estatisticamente para essa propriedade física. Os tratamentos T1-M e T3-M não apresentaram diferença estatística entre si $(p>0,05)$, porém apresentaram diferença estatística $(p<0,05)$ entre T1-M e T2-M e, T2-M para T3-M no MOR direção longitudinal. Na direção transversal, observou-se que o tratamento T2-M se diferenciou dos demais, tanto para o MOR quanto para o MOE. O tratamento com o menor teor de resina (T1-M) implicou em resultados de Al diferentes aos do tratamento T2-M, havendo, porém, o compartilhamento com os resultados do tratamento T3-M.

Embora os tratamentos apresentem algumas diferenças estatísticas, todos atenderam as recomendações da norma EN 300:2002 - Oriented Strand Board (OSB) - Definitions, classification and specifications, painéis do Tipo 1, para as propriedades mecânicas. Assim, o tratamento $\mathrm{T} 1-\mathrm{M}$ (13\% de resina PU de mamona) com a vantagem do menor consumo de resina que impacta no custo de produção de painéis particulados (Barros Filho, 2009), é selecionado como o mais adequado para produção de painéis OSB com partícula de madeira Balsa residual.

Barbirato et al. (2018) apresentou um estudo de painéis de partículas orientadas (OSB) a partir de resíduos de madeira Balsa aglomeradas com resina poliuretana à base de óleo de mamona. Os painéis foram avaliados pelos autores com diferentes densidades $\left(300 \mathrm{~kg} / \mathrm{m}^{3} \mathrm{e}\right.$ $400 \mathrm{~kg} / \mathrm{m}^{3}$ ) em diferentes teores (11\% e 15\%). Os resultados obtidos indicaram que os painéis de $400 \mathrm{~kg} / \mathrm{m}^{3}$ de densidade e $15 \%$ de resina atingiram valores de MOR $(18,72 \mathrm{MPa})$ e MOE (3505MPa) similares ao encontrado no T1-M que os classificaram como OSB tipo 1, segundo recomendações da EN 300:2002. 


\section{Painéis OSB com resina FF}

Os painéis produzidos com os tratamentos de resina FF, também com teores de 13, 15 e $18 \%$, apresentaram conforme Tabela 4, os valores médios dos ensaios físico e mecânicos para 8 repetições das propriedades IE, MOR, MOE e Al. O tratamento T1-FF, apresentou o valor de inchamento $(26,42 \%)$ maior que o limite estabelecido pela norma EN 300:2002, no entanto esse valor é estaticamente igual aos demais grupos que resultaram em valores inferiores a $25 \%$. Isto demonstra que os teores de FF avaliados implicam para essa propriedade física em resultados próximos ao limite preconizado por norma.

Tabela 4. Valores médios das propriedades físicas e mecânicas de painéis OSB com resina FF

\begin{tabular}{ccccccc}
\hline \multirow{2}{*}{ Tratamento } & IE (\%) & \multicolumn{2}{c}{ MOR (MPa) } & \multicolumn{2}{c}{ MOE (MPa) } & \multirow{2}{*}{ Al (MPa) } \\
& 24 horas & Long. & Trans. & Long. & Trans. & \\
\hline T1-FF & $26,42^{\mathrm{a}}$ & $22,51^{\mathrm{a}}$ & $19,69^{\mathrm{a}}$ & $4455^{\mathrm{b}}$ & $1838^{\mathrm{a}}$ & $0,30^{\mathrm{a}}$ \\
(CV) & 11,06 & 15,84 & 13,9 & 17,86 & 18,33 & 33,88 \\
T2-FF & $23,57^{\mathrm{a}}$ & $23,77^{\mathrm{a}}$ & $18,39^{\mathrm{a}}$ & $5881^{\mathrm{a}}$ & $1832^{\mathrm{a}}$ & $0,28^{\mathrm{a}}$ \\
(CV) & 30,82 & 17,71 & 30,55 & 16,72 & 19,95 & 30,64 \\
T3-FF & $23,37^{\mathrm{a}}$ & $25,50^{\mathrm{a}}$ & $15,24^{\mathrm{a}}$ & $5707^{\mathrm{a}}$ & $1742^{\mathrm{a}}$ & $0,29^{\mathrm{a}}$ \\
(CV) & 33,11 & 10,4 & 30,34 & 25,49 & 16,17 & 41,48 \\
EN 300:2002 Tipo 1 & 25 & 20 & 10 & 2500 & 1200 & 0,3 \\
\hline
\end{tabular}

*Médias seguidas por letras diferentes na coluna diferem significativamente a 5\% pelo Teste Tukey

Os tratamentos de $\mathrm{FF}$, no geral, não apresentaram diferenças estatística entre si $(p>0,05)$, com exceção de T1-FF para MOE na direção longitudinal. Todos os tratamentos atenderam as recomendações da norma EN 300:2002 - Oriented Strand Board (OSB) Definitions, classification and specifications, painéis do Tipo 1, para as propriedades mecânicas de MOR e MOE. Já, para a propriedade de Al apresentarem média de T2-FF e T3FF inferiores ao recomendado pela norma, no entanto, por não ser estatisticamente diferente de T1-FF infere-se que esses tratamentos, ainda que no limite de $\mathrm{Al}=0,30 \mathrm{MPa}$, também atenderam as recomendações normativas. Assim, o tratamento T1-FF (13\% de resina FF) é selecionado como o mais adequado para produção de painéis OSB com partícula de madeira Balsa residual.

Bufalino et al. (2015) estudou a viabilidade de painéis OSB produzidos a partir de uma mistura das madeiras Toona ciliata, Eucalyptus grandis/urophylla e Pinus oocarpa na orientação para face/miolo/face e adesivo FF com uma proporção de $9 \%$. A maioria dos painéis OSB misturados não atingiram plenamente aos requisitos especificados pela norma EN 300:2002, para as propriedades físicas e mecânicas. Entretanto, os painéis OSB fabricados com madeiras de Eucalyptus grandis/urophylla apresentaram potencial similar ao encontrado no T1-FF, com valores de 0,35 para Al, $4561 \mathrm{MPa}$ para MOE e 28,2 MPa de MOR, caracterizando como OSB tipo 1, de acordo com as recomendações da EN 300:2002.

\section{Painéis OSB com resina UF}

Os tratamentos de UF, com resultados apresentados para 8 repetições na Tabela 5, mostrou que a variação no teor dessa resina implica em diferenças estatísticas para a maioria das propriedades físicas e mecânicas avaliadas, com exceção de Al. Destaca-se também, que nenhum dos tratamentos desta pesquisa atende na sua totalidade as recomendações da norma EN 300:2002.

No caso da Al das partículas, em todos os tratamentos resultaram valores muito inferiores aos recomendados pela norma EN 300:2002 (Al>0,30). Assim, não se recomenda nenhum dos tratamentos avaliados nesta pesquisa como adequados para a produção de painéis OSB com partículas de madeira Balsa e resina UF. 
Tabela 5. Valores médios das propriedades físicas e mecânicas de painéis OSB com resina UF

\begin{tabular}{|c|c|c|c|c|c|c|}
\hline \multirow{2}{*}{ Tratamento } & \multirow{2}{*}{$\frac{\text { IE }(\%)}{24 \text { horas }}$} & \multicolumn{2}{|c|}{ MOR (MPa) } & \multicolumn{2}{|c|}{ MOE (MPa) } & \multirow{2}{*}{ Al (MPa) } \\
\hline & & Long. & Trans. & Long. & Trans. & \\
\hline T1-UF & $103,61^{c}$ & $3,81^{c}$ & $2,97^{b}$ & $642^{c}$ & $301^{c}$ & $0,02^{a}$ \\
\hline (CV) & 6,15 & 29,26 & 38,95 & 25,39 & 44 & 46,29 \\
\hline T2-UF & $74,21^{b}$ & $9,63^{b}$ & $9,07^{a}$ & $2938^{b}$ & $1016^{b}$ & $0,04^{a}$ \\
\hline (CV) & 9,22 & 17,37 & 24,46 & 26,12 & 30,85 & 23,15 \\
\hline T3-UF & $38,49^{a}$ & $20,82^{a}$ & $14,40^{a}$ & $5825^{a}$ & $1778^{a}$ & $0,03^{a}$ \\
\hline (CV) & 16 & 17,09 & 21,09 & 20,59 & 27,76 & 54,11 \\
\hline EN 300:2002 Tipo 1 & 25 & 20 & 10 & 2500 & 1200 & 0,3 \\
\hline
\end{tabular}

*Médias seguidas por letras diferentes na coluna diferem significativamente a 5\% pelo Teste Tukey

Hellmeister (2017) avaliou painéis OSB, com resíduo de madeira Balsa proveniente de reflorestamento, aglomerados com teor de $8 \%$ de resina UF. Os resultados obtidos pelo autor demonstraram que os painéis OSB não atingiram as recomendações físicas e mecânicas para painéis OSB tipo 1, de uso interno e não estrutural da EN 300:2002.

\section{Características anatômicas dos painéis OSB de madeira Balsa residual}

A Figura 3 apresenta imagens de MEV da seção transversal dos painéis T1-M e T1-FF com uma ampliação de 50x. Nota-se uma adequada compactação das partículas, corroborando com os resultados de $\mathrm{Al}$ dos quais é possível inferir se a quantidade de resina utilizada na produção do painel está adequada. Na Figure 3B, observa-se a existência de (um maior número de) vazios, característica essa que contribuiu para elevar os valores de IE das amostras do grupo de tratamento T1-FF.

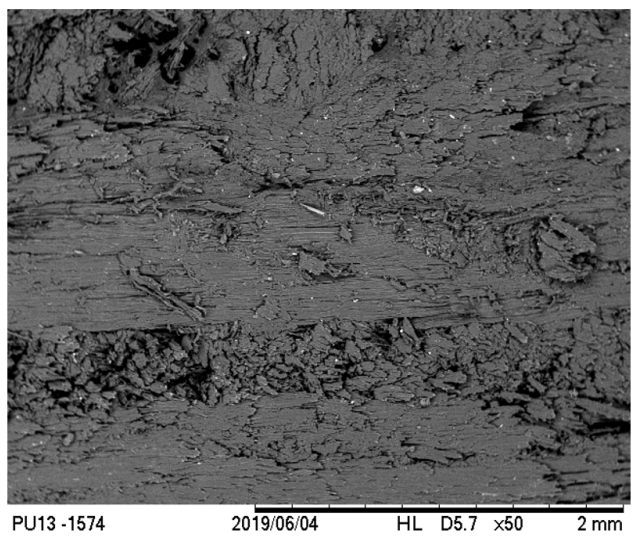

(A)

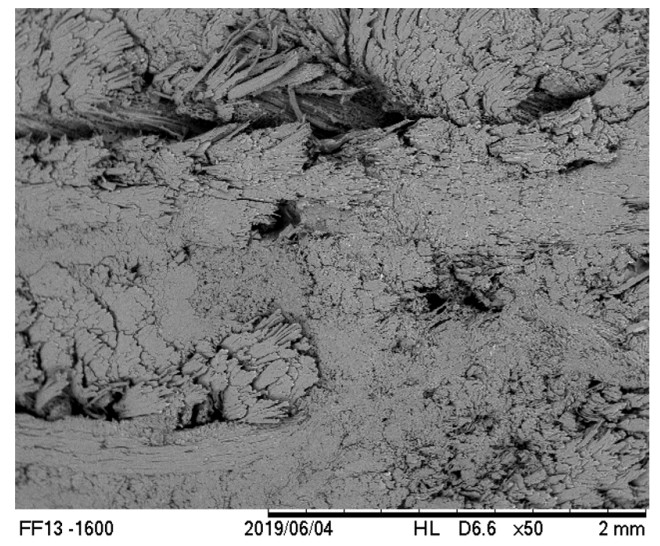

(B)

Figura 3: Seção transversal do painel OSB. a) T1-M. b) T1-FF com aumento de 50x.

\section{CONCLUSÃO}

Pelos resultados das propriedades físicas, químicas, anatômicas e mecânicas o resíduo de madeira Balsa (Ochroma Pyramidale) apresenta potencial para fabricação de painéis OSB.

As variações nas propriedades física e mecânicas em função do teor de resina utilizado na fabricação das placas foram menos significativas para o tratamento FF. Comparativamente, para cada teor de resina analisado verificou-se que a composição com PU de mamona proporcionou painéis com os menores índices de absorção, sendo, portanto, mais estáveis sob aspectos da higroscopicidade. Os painéis OSB produzidos com densidade de $650 \mathrm{~kg} / \mathrm{m}^{3} \mathrm{e}$ 13\% de resina PU de mamona e FF apresentaram propriedades físicas e mecânicas que 
atendem as exigências da norma EN 300/2002 para placas OSB do tipo 1 sendo recomendados para ambientes internos ou para produção de mobiliário.

\section{AGRADECIMENTOS}

Os autores são gratos à FAPESP (proc. 2017/18076-4) e ao CNPq (proc. 407451/2018-8 e proc. 304106/2017-8). “Este estudo foi financiado em parte pela coordenação de aperfeiçoamento de pessoal de nível superior-Brasil (CAPES) - código financeiro 001".

\section{REFERÊNCIAS}

Associação Brasileira de Normas Técnica - ABNT. (1997). NBR 7190:- Projeto de estruturas de madeira. Métodos de ensaio para determinação de propriedades das madeiras para o projeto de estruturas Valores médios usuais de resistência e rigidez de algumas madeiras nativas e de florestamento (107 p.). Rio de Janeiro: ABNT.

Barbirato, G. H. A., Lopes Junior, W. E., Hellmeister, V., Pavesi, M. \& Fiorelli, J. (2018). OSB panels with balsa wood waste and castor oil polyurethane resin. Waste and Biomass Valorization. (11) 743-751.

Barros Filho, R. M. (2009). Painéis aglomerado a base de bagaço de cana-de-açúcar e resinas uréia formaldeído e melamina formaldeído (Dissertação de Mestrado). Curso de Engenharia de Materiais Processos de Fabricação, Departamento de Engenharia de Materiais, Rede Temática em Engenharia de Materiais, Ouro Preto.

Borrega, M., Ahvenainen, P., \& Gibson, L. (2015). Mechanics of balsa (Ochroma pyramidale) wood. Mechanics of Materials, 84, 75-90. http://dx.doi.org/10.1016/j.mechmat.2015.01.014.

Bortoletto Júnior, G., \& Garcia, J. N. (2004). Propriedades de resistência e rigidez à flexão estática de painéis compensados e OSB. Revista Árvore, 28(4), 557-564. http://dx.doi.org/10.1590/S010067622004000400010.

Brito, J. O., \& Barrichelo, L. E. G. (1977). Correlações entre as características físicas e químicas da madeira e a produção de carvão vegetal: I - densidade e teor de lignina da madeira de Eucalipto. IPEF, (14), 9-20.

Bufalino, L., Ribeiro Corrêa, A. A., Sá, V. A., Marin Mendes, L., Amarante Almeida, N., \& Dordenoni Pizzol, V. (2015). Alternative compositions of oriented strand boards (OSB) made with commercial woods produced in Brazil. Maderas. Ciencia y Tecnologia, Concepción, 17(1), 105-116. http://dx.doi.org/10.4067/S0718-221X2015005000011.

Christoforo, A. L., Silva, S. A. M., Barbosa, J. C., Ribeiro Filho, S. L. M., Panzera, T. H., \& Rocco Lahr, F. A. (2015). Produção de chapas de partículas com resíduos da madeira Cordia goeldiana. Engenharia Agrícola, 35(2), 368-377. http://dx.doi.org/10.1590/1809-4430-Eng.Agric.v35n2p368-377/2015.

Cloutier, A. (1998). Oriented strandboard (OSB): raw material, manufcturing process, properties, and uses. In First International Seminar on Solid Wood Products of High Technology (pp. 173-185), Belo Horizonte, MG.

Congresso Internacional de Biomassa. (2018). Mercado e oportunidades da biomassa florestal no Brasil. Retrieved in 2018 October 05, from http://www.stcp.com.br/wpcontent/uploads/2018/09/CIBIO_2018_Mercado_Biomassa_Florestal_FINAL.pdf

Cravo, J. C. M., Sartori, D., Marmol, G., Balieiro, J. C. C., Machado, G. O., \& Fiorelli, J. (2015). Manufacture of particleboard based on cement bag and castor oil polyurethane resin. Construction \& Building Materials, 87, 8-15. http://dx.doi.org/10.1016/j.conbuildmat.2015.03.114.

D’Almeida, M. L. O. (1988). Composição química dos materiais lignocelulósicos. In M. L. O. D'Almeida. Tecnologia de fabricação da pasta celulósica (2. ed.). São Paulo: IPT.

Da Silva, A., \& Kyriakides, S. (2007). Compressive response and failure of balsa wood. International Journal of Solids and Structures, 44(25-26), 8685-8717. http://dx.doi.org/10.1016/j.ijsolstr.2007.07.003.

Daniels, P. L. (2017). Working with Balsawood - The definitive guide to using Balsa. Retrieved in 2017, September 13, from: http://pldaniels.com/flying/balsa/balsa-properties.html

Easterling, K. E., Harrysson, R., Gibson, L. J. \& Ashby, M. F. (1982). On the mechanics of balsa and other woods. Proceedings of The Royal Society A, 383, p. 31-41.

Empresa Brasileira de Pesquisa Agropecuária. (2010). Metodologias para avaliação de alimentos para ruminantes domésticos. Porto Velho, RO: Embrapa Rondônia. 21 p. 
European Committee for Standardization. (1993a). EN 310: Wood-based panels: determination of modulus of elasticity in bending and of bending strength. Bruxelas.

European Committee for Standardization. (1993b). EN 317: Particleboards and fibreboards: determination of swelling in thickness after immersion in water. Bruxelas.

European Committee for Standardization. (1993c). EN 319: Particleboards and fibreboards: determination of tensile strength perpendicular to the piane of the board. Bruxelas.

European Committee for Standardization. (2002). European Standard - EN 300: Aglomerado de partículas de madeira longas e orientadas (OSB) - Definições, classificação e especificações. Portugal.

Fengel, D., \& Wegener, G. (1984). Wood chemistry, ultra strucuture- Reactions. Berlin: Walterde Gruyter.

Finger, Z., Logsdon, N. B., \& Rocha, K. J. (2012). Descrição dendrológica e caracterização física da madeira de Pau-de-balsa, Ochroma pyramidale (Cav. ex Lam) Urb. In Encontro Brasileiro em Madeiras e Estruturas de Madeira. Vitória. UFES. CD-ROM 1.

Fiorelli, J., Gomide, C. A., Lahr, F. A. R., de Lucca Sartori, D., \& Belini, U. L. (2014). Physico-chemical and anatomical characterization of residual lignocellulosic fibers. Cellulose, 21(5), 3269-3277.

Hellmeister, V. (2017). Painel OSB de resíduo de madeira Balsa (Ochroma Pyramidale) (Tese de Doutorado). Faculdade De Zootecnia e Engenharia De Alimentos, Universidade de São Paulo, Pirassununga.

Khalil, A. H. P. S., Fazita, M. R., Bhat, A. H., Jawaid, M., \& Fuad, N. A. (2010). Development and material properties of new hybrid plywood from oil palm biomass. Materials and Design, 31, 417-424.

Macedo, L. B. D., Silva, M. R., César, A. A. S., Panzera, T. H., Christoforo, A. L., \& Lahr, F. A. R. (2016). Painéis OSB de madeira Pinus sp. e adição de partículas de polipropileno biorientado (BOPP). Scientia Forestalis, 44(112), 887-894. http://dx.doi.org/10.18671/scifor.v44n112.10.

Megaton, A. S., Oliveira, R., Lopes, O. R., Milagres, F. R., Piló-Veloso, D., \& Colodette, J. L. (2006). Composição Química da Madeira de Espécies de Eucalipto. In: Anais da 29ª Reunião Anual da Sociedade Brasileira de Química. Águas de Lindóia: SBQ .

Mendes, R. F., et al (2010). Painéis aglomerados produzidos com bagaço de cana em associação com madeira de eucalipto. Scientia Forestalis, Piracicaba, 38(86), 285-295.

Moura, M. J., \& Figueiredo, M. M. (2002). Aplicação das Técnicas de Picnometria de Gás e de Porosimetria de Mercúrio à Caracterização da Madeira de E. globulus. Portugal: LABGRAN. Universidade de Coimbra. Departamento de Engenharia Química.

Ortunõ, T. G., Rodrígues, J. A., García, M. T. F., Villena, M. F., \& García, C. E. F. 2011. Evaluation of the physical end mechanical properties of particleboard made from giant reed (Arundo donax L.). BioResources, 6(1), 477-486.

Ronquim, R. M., Ferro, F. S., Icimoto, F. H., Campos, C. I., Bertolini, M. S., Christoforo, A. L., \& Rocco Lahr, F. A. (2014). Physical and Mechanical Properties of Wood-Cement Composite with Lignocellulosic Grading Waste Variation. International Journal of Composite Materials, 4(2), 69-72. http://dx.doi.org/10.5923/j.cmaterials.20140402.05.

Van Soest, P. J. (1994). Nutritional ecology of the ruminant. New York: Cornell University Press. http://dx.doi.org/10.7591/9781501732355.

Varanda, L. D., Christoforo, A. L., Almeida, D. H., Silva, D. A. L., Panzera, T. H., \& Rocco Lahr, F. A. (2014). Evaluation of modulus of elasticity in static bending of particleboards manufactured with Eucalyptus grandis wood and oat hulls. Acta Scientiarum Technology (Impresso), 36, 405-411.

Vital, B. R. (1973). Effects of species and panel densities on properties of hardwood particleboard. $111 \mathrm{f}$. (Master of Science Forestry) - University of Wisconsin, Madison.

Contribuição dos Autores: WELJ: Conceituação, Curadoria de Dados, Investigação, Metodologia, Administração do Projeto, Recursos, Software, Validação, Visualização, Escrita - Primeira Redação, Escrita - Revisão e Edição. GHAB: Metodologia, Software, Visualização. MP: Curadoria de Dados, Recursos, Software, Visualização. JS: Curadoria de Dados, Análise Formal, Visualização, Escrita - Revisão e Edição. JF: Conceituação, Análise Formal, Obtenção de Financiamento, Supervisão, Validação, Visualização, Escrita - Revisão e Edição. 